Management international

International Management

Gestiòn Internacional

La transition des individus par des épisodes de pauvreté : de l'ajustement de la consommation à la reconfiguration identitaire

The Transition of Individuals Through Episodes of Poverty: From Adjustment of Consumption to Identity Reconfiguration

La transición de los individuos por episodios de pobreza: del ajuste del consumo a la reconfiguración identitaria

\author{
Abdelmajid Amine et Alia Toumi
}

Volume 22, numéro 2, hiver 2018

URI : https://id.erudit.org/iderudit/1058167ar

DOI : https://doi.org/10.7202/1058167ar

Aller au sommaire du numéro

Éditeur(s)

HEC Montréal

Université Paris Dauphine

ISSN

1206-1697 (imprimé)

1918-9222 (numérique)

Découvrir la revue

Citer cet article

Amine, A. \& Toumi, A. (2018). La transition des individus par des épisodes de pauvreté : de l'ajustement de la consommation à la reconfiguration identitaire. Management international / International Management / Gestiòn Internacional, 22(2), 144-158. https://doi.org/10.7202/1058167ar
Résumé de l'article

Cette recherche explore les stratégies d'ajustement de la consommation mises en oeuvre par les individus traversant des épisodes de pauvreté ainsi que les arrangements identitaires à l'oeuvre lors de ces transitions. Nos résultats montrent que ces individus déploient, outre les stratégies d'ajustement mobilisées par les consommateurs " durablement pauvres », des stratégies d'émancipation du marché reposant sur la résistance à la consommation et l'adoption de comportements déviants. Ces adaptations de la consommation se doublent d'accommodements identitaires permettant d'assurer une congruence du soi avec la situation précaire vécue. Enfin, des implications sont dressées en direction du marché, des pouvoirs publics et des organisations de la société civile en vue d'inclure ces consommateurs transitoirement pauvres.
Tous droits réservés (C) Management international / International Management / Gestión Internacional, 2018
Ce document est protégé par la loi sur le droit d'auteur. L’utilisation des services d’Érudit (y compris la reproduction) est assujettie à sa politique d'utilisation que vous pouvez consulter en ligne.

https://apropos.erudit.org/fr/usagers/politique-dutilisation/ 


\title{
La transition des individus par des épisodes de pauvreté : de l'ajustement de la consommation à la reconfiguration identitaire ${ }^{\star}$
}

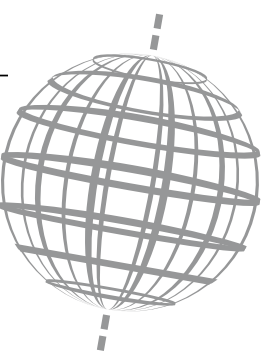

\author{
The Transition of Individuals Through Episodes of Poverty: \\ From Adjustment of Consumption to Identity Reconfiguration
}

\section{La transición de los individuos por episodios de pobreza: del ajuste del consumo a la reconfiguración identitaria}

\author{
ABDELMAJID AMINE \\ IRG - Université Paris-Est
}

\author{
ALIA TOUMI \\ IRG - Université Paris-Est
}

\section{RÉSUMÉ}

Cette recherche explore les stratégies d'ajustement de la consommation mises en œuvre par les individus traversant des épisodes de pauvreté ainsi que les arrangements identitaires à l'œuvre lors de ces transitions. Nos résultats montrent que ces individus déploient, outre les stratégies d'ajustement mobilisées par les consommateurs « durablement pauvres", des stratégies d'émancipation du marché reposant sur la résistance à la consommation et l'adoption de comportements déviants. Ces adaptations de la consommation se doublent d'accommodements identitaires permettant d'assurer une congruence du soi avec la situation précaire vécue. Enfin, des implications sont dressées en direction du marché, des pouvoirs publics et des organisations de la société civile en vue d'inclure ces consommateurs transitoirement pauvres.

Mots-Clés : Episode de pauvreté, reconfiguration identitaire, stratégies d'ajustement, transition, émancipation, consommation.

\section{ABSTRACT}

This research explores the coping strategies implemented by consumers crossing a temporary period of financial restraint. Our findings show that, in addition to traditional adjustment strategies mobilized by permanently poor consumers, individuals facing a temporary precarious situation deploy other strategies allowing them to emancipate from the market based primarily on resistance to consumption and on adoption of deviant behaviors. These adjustments of consumption are coupled with identity arrangements to ensure self-congruence with the experienced precarious situation. Finally, some implications and avenues are drawn for the market, public authorities and civilian society organizations in order to include those consumers temporarily impoverished. Keywords: Episode of poverty, identity reconfiguration, coping strategies, transition, emancipation, consumption.

\section{RESUMEN}

Esta investigación explora las estrategias de ajuste utilizadas por los individuos que atraviesan episodios de pobreza y las reconfiguraciones identitarias que operan durante estas transiciones. Los resultados muestran que estos individuos movilizaron, además de las estrategias de ajuste utilizadas por los consumidores "durablemente pobres", las estrategias de emancipación del mercado basado principalmente en la resistencia al consumo y a la adopción de conductas desviantes. Estos ajustes del consumo se acompañan de reconfiguraciones identitarias que permiten asegurar una congruencia del Yo con la situación precaria vivida. Por último, con el fin de incluir estos consumidores temporalmente pobres, diferentes pistas son formuladas en dirección del mercado, de los poderes públicos y las organizaciones de la sociedad civil.

Palabras Clave: Episodio de pobreza, reconfiguración identitaria, estrategia de ajuste, transición, emancipación, consumo.

\begin{abstract}
Cette recherche explore les comportements des individus ayant vécu un épisode de baisse temporaire des ressources et les stratégies d'ajustement de la consommation qu'ils mettent en place dans cette situation ainsi que les arrangements identitaires qui en résultent. Elle soutient que ce phénomène de pauvreté transitoire a été beaucoup négligé dans les recherches en gestion alors qu'il a tendance à se généraliser et qu'il présente d'importants enjeux économiques et sociétaux.
\end{abstract}

En effet, dans la société de consommation caractérisée par l'abondance des produits, les ressources financières des consommateurs sont naturellement considérées comme un vecteur de marquage identitaire et social et comme jouant un rôle déterminant dans les choix opérés. Toutefois, force est de constater que cet appétit de consommer, habituellement aiguisé par la tentation du marché, se voit remis en cause par l'altération du pouvoir d'achat favorisée par la dernière crise économique et financière qui se distingue par son caractère systémique et touche aux fondements de l'économie de marché (Laville, 2011). Au-delà de sa dimension économique, cette crise s'accompagne d'une défiance grandissante à l'égard des valeurs de l'idéologie de marché dans la mesure où elle alimente un large mouvement de contestation comme en témoignent, en France notamment, les larges franges de la population exposées à des situations de précarité financière et sociale (Tableau 1). Les individus

* Remerciements :

Les auteurs remercient chaleureusement les rédacteurs en chef de la revue Management International ainsi que les deux relecteurs dont les remarques et suggestions ont contribué à améliorer la qualité de cet article. 
concernés se comptent d'abord parmi les seniors vivant d'une retraite insuffisante et qui doivent pour certains reprendre le travail pour compléter leurs maigres revenus. Ensuite, les jeunes, diplômés compris, peinent à accéder au monde du travail et enregistrent un taux de pauvreté atteignant les $20 \%$ chez les 18-24 ans. Sont aussi concernés des cadres basculant dans des situations de chômage de courte ou longue durée, des salariés vivant dans la précarité et des ménages monoparentaux qui survivent grâce à de multiples aides publiques. Les difficultés de fin de mois obligent ces individus fragilisés, qui constituent près de $20 \%$ de la population Française, à faire des arbitrages cornéliens où les dépenses de santé sont généralement sacrifiées en premier (Baromètre Ipsos/Secours Populaire 2016).

Si, par le passé, les individus issus de catégories socioprofessionnelles élevées étaient épargnés par ce type d'incidents, ce n'est plus le cas aujourd'hui, car la baisse des ressources n'affecte plus uniquement une classe d'individus structurellement pauvre ou marginale, mais touche aussi des classes sociales plus aisées. Ce phénomène reflète des situations de «nouvelles pauvretés» (Couret; Metzger, 2009) et révèle que la pauvreté n'est plus une condition permanente mais devient un épisode dans la vie des individus. Par conséquent, les apports des travaux antérieurs sur la pauvreté structurelle doivent être réévalués à l'aune de cette nouvelle donne qui est appelée à perdurer.

En temps de restriction des ressources financières, les aspects de la vie pris pour acquis sont amenés à être réévalués. Ainsi, la recherche auprès des individus en situation de pauvreté temporaire peut nous éclairer, d'un côté sur les manières dont la consommation des biens et services est reconfigurée en vue de s'adapter à la situation de précarité vécue; et de l'autre sur la tendance de ces individus à s'engager dans une réévaluation identitaire à la suite des difficultés rencontrées.

\section{Pauvreté durable $v s$ temporaire du consommateur}

Les années 1960-70 ont donné naissance à ce que Laville (2011) appelle «crise de valeur» qui traduit un «effritement de l'idéologie du progrès » et qui a impulsé une multitude d'innovations ayant trait à l'alimentation, aux énergies renouvelables, au recyclage, etc. En ce temps, la pauvreté ${ }^{1}$ était plutôt «héritée » et "davantage le fait d'une population en marge du progrès social qui perdurait en dépit de la montée de l'État-providence» (Favreau, 2003). Mais grâce au plein emploi, l'accès à la formation et l'essor des services publics entre 1970 et le milieu des années 80 , la pauvreté a grandement baissé. Durant cette période, on s'est écarté des priorités de la décennie précédente et «la pauvreté de crise » s'est installée, poussant les associations à adopter une posture plus «défensive» à l'image de la lutte contre le chômage ou du maintien d'un revenu décent (Laville, 2011). Les années 2000 ont vu les conditions socio-économiques s'aggraver de manière structurelle pour différentes franges de la population confrontées à la montée du chômage, de la pauvreté et de la précarisation des conditions d'emploi et aux difficultés d'accès aux biens publics (éducation, eau, santé, habitat). Cette dégradation des conditions de vie a donné lieu à l'émergence de nouvelles formes de solidarité portées par les organisations de la société civile (Fraisse; Guérin; Laville, 2007).
Si cette évolution donne à voir une photographie de la santé économique des ménages et des mutations de la société, elle ne distingue pas entre les personnes vivant dans une pauvreté chronique qui nécessitent un traitement social spécifique et les individus qui connaissent une pauvreté transitoire suite à un accident de la vie et qui requièrent un autre type d'accompagnement afin d'éviter qu'ils s'installent structurellement dans la pauvreté. En effet, si le phénomène est d'ampleur globale, les pays développés connaissent en particulier depuis les années 2000 de nouvelles formes de pauvreté, de précarité et d'insécurité. Ainsi, des sociologues, tels que Leisering et Leibfried (1999), ont souligné que la pauvreté dans les sociétés contemporaines est principalement un phénomène transitoire et que la frange d'individus touchée par une pauvreté durable devient minoritaire (Paugam; Seltz, 2005).

D'un point de vue pratique, si la distinction entre pauvreté chronique et pauvreté transitoire reste un exercice assez complexe (McKay; Lawson, 2003), la première peut néanmoins être cernée à partir de sa persistance dans le temps (Hulme; Shepherd, 2003). Un individu ou un ménage est structurellement pauvre si, au fil du temps, l'on observe que son bien-être est et reste en dessous de la norme économico-sociale minimale.

En outre, si dans le champ de la consommation les recherches centrées sur les individus structurellement pauvre restent assez rares (i.e. Gorge; Ozçaglar-Toulouse, 2013; Mini-dossiers «pauvreté, précarité et consommation » de la revue Economies et Sociétés, 2014/2015) ou généralement adossées aux travaux sur le prix consenti, le type de produits achetés ou les lieux d'achat fréquentés, ce n'est pas le cas d'autres disciplines en sciences sociales qui se sont emparées depuis longtemps du thème de la pauvreté à l'instar de l'ouvrage séminal de Simmel (1907/1998) ou des travaux menés par Caplovitz (1963). Dans ces recherches,

\begin{tabular}{|l|l|}
\hline \multicolumn{2}{|c|}{ QA BLEAU 1 } \\
entre 2012 et 2016 \\
\hline Indicateurs de pauvreté en France & $\begin{array}{l}\text { Pourcentages } \\
\text { et chiffres }\end{array}$ \\
\hline Taux de pauvreté (2014) & $14,2 \%$ \\
\hline Nombre de pauvres (2013) & 8,5 millions \\
\hline Nombre de personnes mal logées (2016) & 3,8 millions \\
\hline Nombre de travailleurs pauvres (2012) & $\begin{array}{l}\text { Entre 1,9 et } \\
3,3 \text { millions }\end{array}$ \\
\hline Nombre de chômeurs (2015) & 2,9 millions \\
\hline Taux de pauvreté chez les 18-24 ans & $20 \%$ \\
\hline $\begin{array}{l}\text { Nombre de personnes couvertes par les } \\
\text { minimas sociaux (2012) }\end{array}$ & 6,3 millions \\
\hline Nombre de ménages surendettés (2012) & 765000 ménages \\
\hline $\begin{array}{l}\text { Taux de personnes renonçant aux soins } \\
\text { médicaux (2016) }\end{array}$ & $20 \%$ \\
\hline $\begin{array}{l}\text { Nombre de personnes retraitées ayant } \\
\text { repris le travail (2012) }\end{array}$ & $\begin{array}{l}500000 \\
\text { personnes }\end{array}$ \\
\hline Sources: Insee, Gecodia Pole Emploi, Barometre & \\
\hline
\end{tabular}

Sources : Insee, Gecodia, Pôle Emploi, Baromètre Ipsos, Le Monde 
la dimension économique de la pauvreté prime dans la définition de l'individu pauvre notamment à travers les notions d'aide, d'assistance sociale dont il bénéficie ou des barrières économiques qu'il rencontre quant à l'accès à la sphère de la consommation. Cette conception de la pauvreté a été critiquée car elle ne prend en considération que les besoins physiques et ne tient pas compte des besoins psychologiques et sociaux (Bauman, 2005). Partant de là, la pauvreté a été comprise comme l'absence de ressources (y compris sociales) ou comme le résultat de l'incompétence des individus dans leur maniement. Cette position, intéressante au demeurant, dépasse le cadre de l'absence des ressources (économiques, sociales ou culturelles) pour inclure la capacité de l'individu à les mobiliser lorsqu'elles sont disponibles. Cette idée converge avec la théorie des capabilités de Sen (1985) dont le principe central est que, afin de fonctionner efficacement dans la société et d'élargir leurs choix de vie, les individus ont besoin de disposer d'un assez large éventail de capabilités et non seulement d'un revenu suffisant.

Les travaux sociologiques se sont ensuite intéressés à de nouveaux champs notionnels comme l'exclusion, la marginalisation ou la disqualification sociale afin de rendre compte des processus de désocialisation des individus pauvres. Ces travaux ont mobilisé d'autres concepts consubstantiels de la pauvreté comme la précarité et la vulnérabilité pour éclairer ce phénomène dans toutes ses facettes. La précarité peut être définie comme «l'absence d'une ou plusieurs des sécurités permettant aux personnes et aux familles d'assumer leurs responsabilités élémentaires et de jouir de leurs droits fondamentaux» (Loisy, 2000; p. 39). Cette définition large ouvre sur une grande variété de situations de fragilité économique et sociale qui, lorsqu'elles sont concomitantes, tendent à déboucher sur des cas d'extrême pauvreté. Ainsi, «les individus sujets aux ruptures sociales ou familiales ainsi qu'à un enchaînement de situations de vie difficiles résultant de ces ruptures (précarité du logement, perte d'emploi, etc.), s'exposent au cumul des différents facteurs de risque liés notamment à la santé, qui favorisent le développement ou l'aggravation des maladies» (Cambois, 2004).

La vulnérabilité est quant à elle conçue comme une double fragilisation (Castel, 1991). D’une part, il y a la précarisation des statuts dans le domaine de l'emploi avec l'accroissement du travail temporaire ou précaire et du chômage qui conduisent à une «désaffiliation" des individus; d'autre part, il y a la fragilisation des «liens sociaux primaires » due à l'instabilité familiale (Castel, 1995). Cette lecture permet de mieux comprendre la montée de la pauvreté et des conditions de vie marquées par l'incertitude du lendemain où l'une des fragilités peut engendrer l'autre et réciproquement. La vulnérabilité découle donc de l'évolution défavorable de la situation socio-économique des individus qui contraint à des ajustements et des recompositions qui touchent à la fois à la consommation et aux composants identitaires et qui s'articulent autour des représentations du passé et du devenir. La vulnérabilité est ainsi entendue comme l'instabilité ou la fragilité de l'insertion de l'individu dans les champs de l'intégration sociale et de la répartition des ressources. Elle entraîne les individus qui se trouvent momentanément dans des difficultés économiques dans un processus de disqualification sociale conduisant progressivement ces derniers à se définir négativement et à devenir en rupture avec les normes sociales. En effet, dans les sociétés marquées par la culture de consommation, la non-participation aux «normes et rituels de consommation » est un élément clé de l'exclusion sociale (Burchard et al. 1999).

Il convient d'admettre à ce stade qu'il demeure difficile de donner une définition absolue et universelle de la pauvreté en raison de sa dynamique, de la multiplicité de ses dimensions et de l'absence d'homogénéité des profils et trajectoires d'individus qui composent la population pauvre. Cependant, Paugam (2005) dresse une typologie intéressante de la pauvreté qui distingue trois formes élémentaires : la pauvreté intégrée (internalisée par les individus, transmissible entre générations et caractérisant davantage des sociétés peu développées sur le plan économique), la pauvreté marginale (caractérisant des groupes sociaux réduits, marginalisés et largement stigmatisés dans des sociétés développées) et la pauvreté disqualifiante (correspondant à l'appauvrissement des individus suite à une baisse brusque du pouvoir d'achat ou au basculement dans le chômage dans des sociétés plutôt prospères). Les deux premières formes renvoient davantage à la pauvreté permanente (héritée ou choisie) alors que la troisième constitue le terreau de la pauvreté temporaire qui nous intéresse en particulier dans cette recherche.

\section{Cadrage théorique de l'étude de la pauvreté transitoire}

Aborder la pauvreté uniquement dans une perspective de permanence comme l'ont fait les travaux antérieurs, ne rend compte ni de l'importance croissante des formes temporaires de pauvreté auxquelles sont confrontées nos sociétés, ni des nouvelles problématiques qu'elles génèrent à l'instar des transitions récurrentes entre des statuts différents et des réajustements de la consommation et des identités.

Dans ce sens, la temporalité de la pauvreté du consommateur, qui en fait plus un épisode de la vie des individus qu'une condition permanente (Toumi, 2015), souligne la pertinence des cadres théoriques de la «transition liminale» (Van Gennep, 1909) pour rendre compte des rites de passage opérés par les individus qui traversent un épisode de pauvreté; et du «coping» (Lazarus, 1966) pour traduire les ressorts et les stratégies mobilisées par ces mêmes individus en vue d'ajuster leurs comportements de consommation aux contraintes imposées par la baisse transitoire des revenus. Ces deux cadres se nourrissent l'un l'autre en s'articulant autour de l'identité de l'individu et renvoient à la capacité de l'expérience de pauvreté temporaire à bousculer celui qui la traverse sur le plan identitaire et à questionner sa consommation.

\section{LA RESTRICTION TEMPORAIRE DES RESSOURCES VUE COMME UNE TRANSITION LIMINALE}

Le passage par une période de baisse temporaire des ressources traduit une transition «liminale» (Van Gennep, 1909) dans la mesure où elle s'accompagne d'un ensemble de rites de passage des situations avant, pendant et après restriction. Lorsqu'elle est vécue brutalement, cette transition s'accompagne d'une forte reconfiguration identitaire en vue de retrouver un nouvel équilibre entre le soi de l'individu et la situation socio-économique expérimentée. Cette notion de rite de passage est conçue par cet auteur comme "un changement dans un des rôles dominants d'un individu marqué par une période transitionnelle ou liminale», 
qui se produit en trois phases successives. Tout d`abord, il y a la phase de séparation où «lyêtre est séparé du cours ordinaire des choses " associées à l'ancien rôle ou statut; vient ensuite la phase liminale où «il mène une existence marginale» qui se traduit par une instabilité et une ambivalence identitaires; et enfin la phase d`agrégation où "l'être se trouve réintégré dans la vie normale avec un statut nouveau» (Figure 1). Ainsi, des événements tels que le divorce, le décès du conjoint, le chômage ou le déclassement social impliquent souvent des pertes et des ruptures importantes et placent les personnes concernées dans des états liminaux par rapport à leur vie personnelle et leurs rôles économiques et sociaux d'avant. Dans ces situations, l'estime de soi et l'identité des individus sont menacées, ce qui incite ces derniers à tenter de préserver les structures qui garantissent la continuité de leurs modes de vie.

Dans le contexte de notre recherche, la phase de transition liminale se confond avec la période de baisse des ressources financières et contraint les individus à une réévaluation identitaire qui les incite à reconsidérer leurs pratiques de consommation en fonction du degré d'altération de leurs positions socio-économiques antérieures. Nous considérons que pendant la période de restriction financière, l'individu sera dans une phase de marge (liminalité) au cours de laquelle les hiérarchies et les repères d'avant sont bouleversés (Van Gennep, 1909) et de nouveaux arbitrages sont opérés en matière de consommation en vue de maintenir ou de restaurer son image de soi. Au sortir de la période de restriction, il sera dans une phase de réintégration sociale (agrégation), qui s'accompagne d'une réévaluation à la fois du sens associé à la consommation et de l'identité de manière consonante avec le nouveau rôle social.
Ces phases de transitions liminales traduisent des passages accompagnés de ruptures sur les plans identitaire, comportemental et affectif. Afin d'adoucir et de résorber les effets inconfortables, voire stigmatisants, de ces ruptures, les individus sont amenés à user de stratégies d'ajustement de leur mode de vie et d'arrangements de leur identité en vue de s'adapter au nouveau contexte.

\section{L'AJUSTEMENT DES PRATIQUES DE CONSOMMATION DURANT L'ÉPISODE DE PAUVRETÉ}

Une réduction des ressources financières s'accompagne généralement d'un réajustement du mode de vie de l'individu en vue de répondre plus ou moins convenablement à ses besoins sous contraintes. Les individus se voient ainsi dans l'obligation de reconsidérer leurs référentiels de consommation lorsqu'ils sont confrontés à une baisse des ressources et vivent cette situation comme une remise en question identitaire causant de la tension et de l'anxiété. D’ailleurs, des études en psychologie montrent que les événements dramatiques de la vie, à l'instar de la perte des ressources, peuvent engendrer un stress pour l'individu (Lazarus, 1966). Ces travaux donnent sens à la mobilisation de la théorie du coping en vue de comprendre l'ajustement des pratiques de consommation dans les situations anxiogènes d'altération des ressources (financières, sociales). Introduit par Lazarus (1966), le concept de coping est entendu comme l'ensemble des «efforts cognitifs et comportementaux faits pour maitriser, tolérer ou traduire les demandes et conflits internes ou externes", Coyne et al. (1981) complètent cette définition pour y inclure la dimension des ressources de l'individu, nécessaire pour rendre compte de la notion de stress. Ils conceptualisent

\section{FIGURE 1 \\ Phases de transition liminale des consommateurs durant l'épisode de pauvreté et incidence sur le plan identitaire}

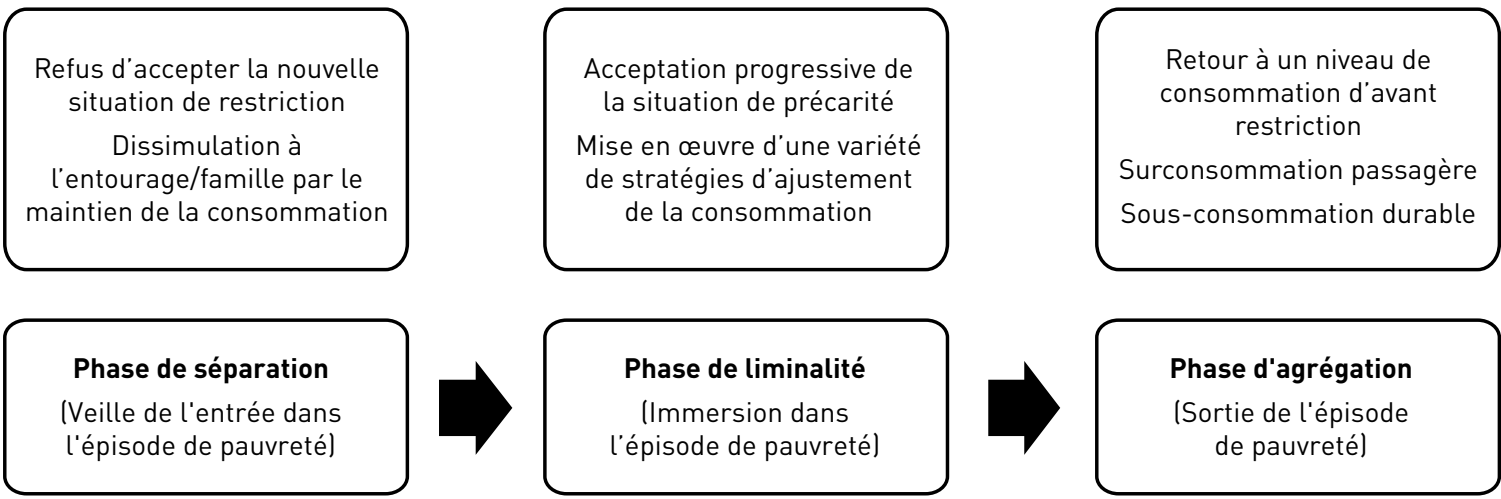

Estime de soi et identité des individus disputées et menacées

Réévaluation identitaire et recherche d'un nouvel équilibre 
dès lors le coping comme l'ensemble des «efforts cognitifs et comportementaux mobilisés pour gérer les conflits et les demandes internes et provenant de l'environnement qui affectent un individu et dépassent ses ressources".

L'apport de la théorie du coping est manifeste pour identifier et comprendre les mécanismes mobilisés par les personnes transitant par un épisode de pauvreté en vue d'ajuster leurs modes de consommation à cette situation génératrice de perte de repères, de stress, et d'altération de l'estime de soi.

\section{LA CONSOMMATION, VECTEUR DE (RE)CONSTRUCTION IDENTITAIRE AU COURS DE L'EXPÉRIENCE DE PAUVRETÉ}

Avec la société de consommation, l'acte de consommer prend une dimension symbolique, idéologique et politique en plus de son ancrage fonctionnel. Dès lors, l'identité des individus peut s'exprimer à travers des actes de consommation dans le sens de "la capacité de se mettre en récit à travers des choix de consommation" (Marris, 1974). Dans la même veine, Goffman (1973) considère que les pratiques de consommation mettent en jeu un ensemble d'interactions dans lesquelles l'individu se met en scène et négocie en permanence son identité qui correspond à une "présentation de soi dans les interactions". Ainsi, les individus s'arrangent avec la culture matérielle pour façonner en permanence leur identité, ce que souligne bien Baudrillard (1988) en disant que nous avons atteint le point où «la consommation a saisi l'ensemble de la vie, où toutes les activités sont devenues mixtes, massées, acclimatées, contrôlées et domestiquées dans la simple activité de shopping perpétuel».

Partant, les pratiques de consommation permettent aux individus d'étendre le champ de leur soi et de mettre en scène des fragments de leur identité. Ainsi, Morin (1969) note que «l'objet et l'usager s'utilisent mutuellement et se modifient l'un par l'autre dans la plus étroite synchronie. La propriété peut ainsi être conçue comme un prolongement de l'être". De ce fait, le concept de soi se voit enrichi à travers le transfert de significations acceptées socialement du produit ou de la marque vers la personne. La consommation permet ainsi aux individus d'exprimer leur identité notamment à travers des logiques d'extension de soi (Belk, 1988). En ce sens, les possessions matérielles peuvent revêtir une signification symbolique en ce qu'elles influencent la manière dont les individus se perçoivent et perçoivent les autres. Les objets consommés ou possédés agissent ainsi comme des signes essentiels à la création et au maintien de l'identité de l'individu et dont la perte ou la privation génère de la peine.

De manière plus prosaïque, les individus qui transitent par un épisode de pauvreté sont amenés, pendant et après cette transition, à procéder à des arbitrages en matière de consommation qui s'accompagnent d'arrangements identitaires en vue de s'adapter à ces situations qui mettent à mal les composants de leur soi individuel et social. Des travaux ont montré que la construction et la préservation de l'identité à travers la consommation se manifestent d'une manière plus prononcée lorsque les individus se trouvent dans des états transitionnels ou liminaux (Schouten, 1991).

\section{Méthodologie de la recherche}

Cette recherche est de nature compréhensive dans le sens où elle s'intéresse à la manière dont les individus transitant par un épisode de pauvreté perçoivent leur réalité de pauvres, les tensions qu'elle institue et les stratégies mises en place pour les résoudre ainsi que les ajustements identitaires qui s'opèrent durant et suite à cette expérience. L'approche qualitative retenue est particulièrement indiquée pour étudier un sujet aussi sensible et empreint d'a priori et de stigmatisation que ne l'est la pauvreté. La réalisation des entretiens s'est effectuée en instaurant une dynamique conversationnelle impliquant le répondant en vue de favoriser un discours tout en profondeur et d'éviter de générer de simples réponses mécaniques.

La mise en œuvre d'un protocole de collecte des données permettant d'explorer le sens de la consommation des personnes transitant par un épisode de pauvreté et les ajustements identitaires qui en découlent, nécessite de délimiter au préalable les contours de la population à étudier tant les situations couvertes par la pauvreté sont diverses et variées. Rentrent ainsi dans le périmètre de notre recherche les individus vivant ou ayant traversé une période de pauvreté caractérisée par une altération des ressources financières par rapport à leur situation d'avant et s'accompagnant ou non d'autres ruptures au niveau social et/ou identitaire (divorce ou séparation, maladie, perte de logement, chômage, etc.). Eu égard au caractère sensible du sujet traité, l'accès aux personnes ayant vécu ce genre d'expérience et répondant aux critères retenus s'est avéré difficile. Par conséquent, nous avons commencé par interroger des personnes de notre entourage qui ont vécu ou qui vivent une situation de difficulté financière et sociale; le recrutement des répondants suivants s'étant opéré de proche en proche en veillant à limiter le risque d'homogénéité des profils et des trajectoires.

$\mathrm{Au}$ final nous avons interrogé 15 personnes (cf. Annexe 1) ayant expérimenté une situation de baisse temporaire des ressources et assez diversifiées en termes d'âge, de sexe, de profession et de durée de l'épisode de pauvreté (2 mois à 2 ans). Cette variété de profils est cruciale dans le sens où les arbitrages en matière de consommation sont en partie déterminés par les conditions initiales et actuelles dans lesquelles vivent ces personnes. Nous avons par ailleurs introduit de la variété en retenant des personnes qui vivaient une période de restriction financière au moment de l'étude (phase de liminalité) et des individus qui l'ont vécu dans un passé récent (moins de six mois) et qui ont pu sortir de cette expérience et investir de nouveaux rôles et statuts sociaux (phase d'agrégation).

Les entretiens d'une durée moyenne de 53 minutes ont généré après retranscription un total de 104 pages times 12 , interligne simple. Ces entretiens ont été réalisés à partir d'un guide peu formalisé en vue de s'adapter à la variété des situations vécues. Les informants étaient invités en préambule à se présenter et à décrire librement leur trajectoire socio-économique de l'avant, pendant et après épisode de pauvreté (pour ceux qui s'en sont sortis). Ensuite les thèmes suivants étaient abordés : (i) le récit $d u$ vécu de la période de précarité, (ii) les stratégies d'ajustement de la consommation mises en place et les arrangements identitaires générés, (iii) les arbitrages opérés en matière de consommation et (iv) les pratiques de consommation post-épisode de pauvreté (vécues ou en projection selon la phase de transition où se trouve le répondant). 
Les entretiens ont été enregistrés à l'aide d'un dictaphone et retranscrits le jour même de l'entretien afin de rester le plus fidèle possible aux discours et conditions du déroulement de l'entretien. Lorsque, dans de rares cas, l'enregistrement gênait le répondant, nous décidions de prendre des notes manuelles afin de le mettre à l'aise et d'aller plus loin dans l'exploration du sujet. Ensuite, les données collectées ont été soumises à une analyse de contenu thématique mobilisant la méthode du codage a posteriori dont l'objectif est de faire émerger à partir des données, des concepts observables en relation avec la situation de précarité temporaire vécue. Dans ce sens, plusieurs itérations de lecture des textes retranscrits ont été effectuées par les auteurs afin de s'approprier le contenu des discours et d'articuler les unités de sens qui se dégagent entre elles avant de les relier à des catégories théoriques signifiantes. Un double codage a été opéré séparément par les auteurs en vue de s'assurer de la fiabilité des interprétations, les divergences rencontrées ayant donné lieu à discussion et à argumentation entre les deux codeurs jusqu'à l'obtention d'un consensus. Les unités d'analyse retenues sont heuristiques car elles aident à faire sens. Elles ont donné lieu par regroupements selon leur proximité de signification à des catégories de sens qui rendent compte d'une part des stratégies mobilisées par les individus pour ajuster leurs pratiques de consommation à la situation de baisse des ressources, et d'autre part, des arrangements identitaires qu'ils ont opérés lors de la transition par l'épisode de pauvreté.

\section{Analyse et discussion des résultats}

Cette recherche a permis d'identifier de nombreuses stratégies de coping et d'ajustement identitaire mises en place par les répondants; certaines reflétant une sorte d'internalisation et d'acceptation de la situation par les individus; et d'autres traduisant des changements transitoires ou structurels affectant la consommation et sa symbolique à l'instar des comportements de déviance et de résistance à (par) la consommation et qui renvoient à des pratiques d'émancipation du marché ou de marginalisation des individus.

Les résultats obtenus sont discutés en lien avec le cadre théorique des transitions liminales et en articulant les stratégies de coping à l'œuvre à l'entrée, durant et après l'épisode de pauvreté aux dimensions identitaire et consommatoire des individus (cf. Figure 2 pour une synthèse des résultats).

\section{RÉSISTANCE «PAR» LA CONSOMMATION LORS DU BASCULEMENT DANS LA PAUVRETÉ}

Dès la phase de séparation avec l'ancien rôle et/ou statut, les prémisses d'une résistance "par» la consommation apparaissent chez les consommateurs qui viennent de verser dans une période de baisse des revenus au travers de leur inclination à ne pas accepter leur nouvelle situation économique et sociale et à nier cette réalité en la dissimulant à leur entourage; à l'image de Heykel (26 ans) : "je disais jamais que j'avais pas, que j'avais des soucis, mais les gens ils voyaient, mais ils me disaient genre allez on va au cinéma, et moi je disais non je suis fatigué j'ai un rendu demain, ils me disaient mais tu te fous de nous, on a le même rendu tous et je rétorquais non, non, je suis fatigué, je dois voir ma mère elle est malade, j'inventais des histoires ... ». On assiste ainsi à une sorte de maquillage de la réalité en vue de préserver son image de soi d'avant. Par ailleurs, sur le plan de la consommation, ces individus tendent à maintenir un niveau de qualité comparable à celui d'avant restriction quitte à en consommer moins, exprimant ainsi, vis à vis de soi et des autres, un rejet de la situation de précarité en continuant à consommer "comme avant». Alexandre (25 ans) étaye bien cette idée; "Pour moi quand j'achète quelque chose où entre guillemets j'investis dans la marque c'est un plaisir ... psychologique je pense. En tout cas oui je prends plus de plaisir à consommer de la marque. Donc pour certaines choses, j'ai continué à acheter de la marque».

En outre, le consommateur temporairement pauvre tend à valoriser les dimensions hédoniques et symboliques associées à la consommation en vue de prolonger la situation précédant l'épisode de pauvreté. Cela traduit à la fois une certaine nostalgie de cette période d'aisance et constitue un sursaut identitaire signifiant que l'individu n'a pas encore accepté de se séparer de l'ancien statut ni de faire le deuil de l'ancien "soi». A titre d'exemple, la consommation de certains loisirs, vue comme «un facteur d'inclusion sociale» (Halbwachs, 1994) n'a, pour certains répondants, été ni arrêtée, ni réduite car elle représente pour eux un important vecteur de socialisation et de préservation de l'image de soi. "Les loisirs ça n'a pas changé, c'est très important. Ça te permet de voir les amis qui en plus vont t'aider à dépenser... parce que ne pas consommer ça peut te 'dé-sociabiliser' à un moment ou à un autre... Donc c'est vital. »(Camille, 31 ans).

Il ressort ainsi que même lorsqu'ils traversent une phase de baisse des ressources, les individus tendent à maintenir une consommation hédonique, parce qu'elle permet de sécuriser l'environnement, de ré-enchanter le quotidien et de sauvegarder l'identité désirée d'avant l'épisode de pauvreté, au détriment d'un basculement vers le tout utilitaire. Cet arbitrage contreintuitif peut être vu comme une sorte de résistance "par» la consommation à la renégociation identitaire que leur imposent leurs nouvelles conditions socio-économiques. Il s'agit d'une lutte, animée par le rejet de l'identité «indésirable» du pauvre en vue de préserver l'identité d'avant via le maintien de certains registres de la consommation.

Par conséquent, la prise de conscience d'être davantage soumis au regard de l'autre lors du basculement dans la période de pauvreté, pousse les individus à se penser, encore plus qu'avant, à travers ce qu'ils consomment, et à s'inscrire dans une sorte d'inclusion des produits consommés ou possédés dans la définition de soi. Cette perspective de préservation identitaire rencontre la notion de «soi étendu» (Belk, 1988) qui élargit le périmètre de l'individu aux objets considérés comme structurants dans le récit de soi. C'est ce qu'exprime Mathilde (39 ans) en disant, "Je pense que la phase de restriction budgétaire m'a poussé à faire plus attention à ce que je consomme en ce sens que c'est un peu "dis-moi ce que tu consommes je te dirai qui tu es", enfin moi perso y'a des fois où je regarde ce que les gens achètent dans leur caddie et j'ai l'impression que ça va me donner un peu une idée sur la manière avec laquelle ils vivent... alors tu vois ils vont acheter du choco et une boite de Nesquick ils ont des enfants tu vois".

Les possessions et les produits consommés sont ainsi considérés comme un reflet de qui nous sommes et sont utilisés comme des marqueurs sociaux. Hoge et Mc Carthy (1984), concluent que ces objets agissent comme des rappels et des confirmateurs 
de l'identité, laquelle peut souvent résider davantage dans ces objets que dans l'individu. En période de transition, la consommation joue un rôle crucial de réassurance, aide à améliorer les sentiments psychologiques de bien-être pendant la phase de liminalité (Noble; Walker, 1997) et contribue à « sauver la face» (Goffman, 1973) en assurant une certaine continuité de soi.

\section{INTERNALISATION DU DÉCLASSEMENT ET ADAPTATION AUX CONDITIONS DE VIE DU « PAUVRE»}

Malgré la résistance par la consommation qu'ils manifestent (cf. supra), les individus qui traversent une phase de pauvreté transitoire finissent, lorsque l'épisode perdure, par internaliser leur nouvelle situation de "pauvre» en déployant des stratégies d'ajustement fondées sur le prix (i) et en activant les registres des relations sociales (ii) en vue de tempérer le poids du déclassement et d'alléger l'acuité des privations subies.
Les stratégies de coping basées sur la maîtrise des dépenses

Elles sont récurrentes dans la littérature sur la pauvreté durable et soulignent une inclination naturelle des pauvres à aller vers le moins cher (Yavas; Riecken, 1981). Mais elles se déclinent dans les discours de nos répondants expérimentant un épisode de pauvreté en une diversité de formes.

Une première stratégie consiste pour certains informants à tenter de satisfaire leurs besoins tout en s'accommodant de la situation de restriction budgétaire par le biais de la planification de leurs dépenses : «Donc j'essayais de prévoir au maximum... pas de dépenses inutiles, pas de dépenses que j'avais pas programmées, ... si je vais acheter un truc c'est que je l'ai déjà programmé minimum une semaine à l'avance » (Heykel, 26 ans). Cela passe aussi par une remise en question de certains modes de paiement en privilégiant notamment les achats en espèces aux paiements par cartes bancaires en vue de maîtriser les dépenses, comme l'illustre bien Karen (22ans) : "Déjà consommer avec la carte

\section{FIGURE 2}

\section{Stratégies de coping avec la consommation durant l'épisode de pauvreté et réarrangements identitaires}

Phase de séparation

(Deuil de l'ancien rôle ou statut)]

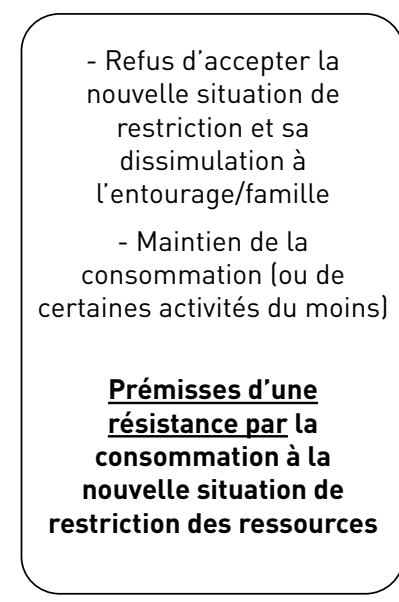

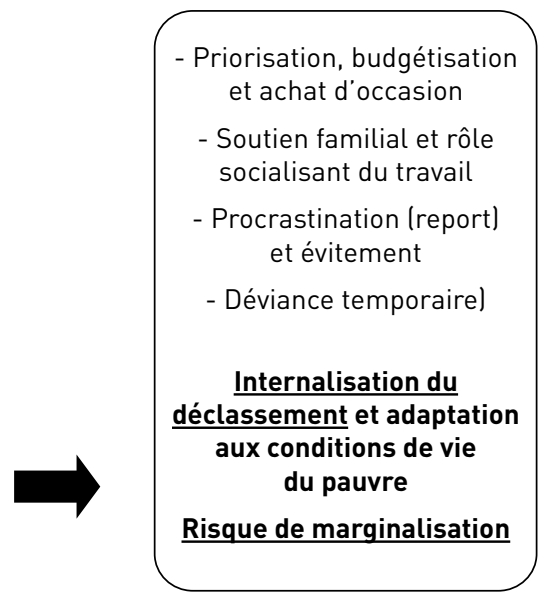

Phase de liminalité

(Flottement identitaire Perte de repères)

Arbitrages basés sur des valeurs symboliques, hédoniques et accessoirement économiques (prix)

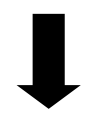

Refus de se séparer de ses anciens statut et identité Tentatives de préserver son image de soi

Rejet puis acculturation progressive avec l'identité du pauvre

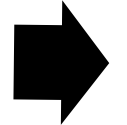

Phase d'agrégation

(Intégration du nouveau rôle ou statut]

- Prise de conscience de la possibilité de consommer autrement

- Résistance durable à la consommation

- Simplicité volontaire (réduction et maîtrise de la consommation]

Réduction volontaire de la consommation

Emancipation choisie du système marchand et de la culture matérielle

Arbitrages fondés sur des valeurs idéologiques

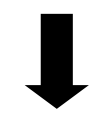

Endossement d'identités nouvelles résistantes et anti culture de consommation 
de crédit ça, j'essaye d'éviter énormément parce que c'est ça qui ruine en fait, c'est la carte de crédit... et j'essaye de retirer beaucoup moins, de payer en liquide car au moins je sais ce que je dépense, je ne tape pas quatre chiffres dans une machine et c'est fini ». Par ailleurs, la maîtrise des dépenses en période de restriction financière incite les individus à expérimenter de nouvelles formes de consommation fondées sur les prix à l'instar de l'achat d'occasion, la recherche des bonnes affaires et le bricolage de solutions alternatives peu coûteuses : "Je suis un peu spécifique, c'est que je n'achète plus les ordinateurs tout faits mais je les achète (en pièces détachées), je les assemble moi-même ça coûte moins cher et finalement j'adore faire ça" (Laurent, 40 ans). La vulnérabilité financière rend ici compte d'un consommateur qui fait attention au prix à travers les canaux classiques de budgétisation et d'achat d'occasion, mais qui est aussi capable de développer une créativité lui permettant de bricoler des solutions originales pour consommer moins cher.

Une autre logique d'économie mobilisée par nos informants consiste à hiérarchiser les dépenses de sorte que l'ajustement de la consommation (alimentation, santé, habillement) s'opère de manière asymétrique dans le foyer. En faisant porter l'effort de restriction sur les parents, ces derniers préservent la consommation des enfants en vue de les protéger des risques de stigmatisation ou d'exclusion par leurs pairs. Cela se traduit par le maintien d'une consommation de qualité pour leur progéniture; "Les vêtements j'y accorde assez d'importance, pour les enfants. Je me suis avisée une fois d'acheter un vêtement à mon fils de la fripe, ça l'a suivi pendant des années, on l'appelait le "sans marque» et on lui demandait s'il était pauvre à l'école ... Depuis je ne le fais plus, même si on a des pépins je ne le fais plus.» (Mathilde, 39 ans). Ces arbitrages dévoilent la tendance des individus à masquer leur pauvreté vis à vis de l'extérieur, en particulier en vue de protéger leurs enfants de la stigmatisation, et rendent compte de la forte pression sociale à laquelle ils sont exposés pour se conformer aux normes du groupe (Hamilton; Catterall, 2006). En raison de leur vulnérabilité, les enfants sont encore plus imprégnés de la culture des marques alimentée par les médias et les groupes de pairs. Des recherches ont d'ailleurs montré l'existence de cette emprise de la société de consommation y compris chez les enfants issus de familles pauvres. Ces derniers s'habillent avec des vêtements de marques et considèrent que, du fait d'être bien habillés, ils «ne peuvent pas» être pauvres (Elliott; Leonard, 2004). Ce déni du statut de "pauvre» traduit une stratégie de défense visant à se mettre à l'abri de la critique et du jugement de l'autre.

\section{Les liens sociaux comme amortisseur des chocs subis}

Outre les stratégies de réduction des dépenses, les consommateurs transitant par un épisode de pauvreté mobilisent leurs relations sociales en tant qu'amortisseur psychologique et financier pour parer aux nouvelles conditions de vie du "pauvre». En effet, de nombreux individus interrogés s'investissent dans le resserrement des liens sociaux parce qu'ils renforcent la capacité à faire face aux difficultés auxquelles ils sont confrontées. Ces relations sociales se cristallisent autour de la famille et des amis d'un côté, et autour des relations de travail pour les actifs, de l'autre. Ainsi, des interviewés ont mentionné l'importance des relations familiales et amicales durant la phase de restriction financière comme un rempart face au décrochage social comme en témoigne Catherine (28 ans) : "J'ai reconnu effectivement mes amis à ce moment-là ainsi que ma famille et effectivement on sent qu'on est jamais seuls dans des moments difficiles et ça c'est très important».

A ce propos, Duhachek (2005) a identifié trois façons dont le soutien social peut être utilisé comme une stratégie d'adaptation. Tout d'abord, le soutien affectif consiste à utiliser les « autres» pour améliorer l'état mental ou émotionnel de l'individu; ensuite, le soutien matériel consiste à obtenir l'aide des autres pour agir directement sur la situation stressante et; enfin, le fait de se ménager émotionnellement et financièrement en se reposant sur l'entourage, permet de récupérer et de mieux s'assumer psychologiquement par la suite.

D'autres répondants ont su, par ailleurs, relativiser la situation vécue en soulignant le caractère structurant et valorisant de la valeur travail et le rôle d'inclusion sociale des relations au travail. Ce faisant, ces individus arrivent à tempérer l'effet de cette situation en préservant une image de soi personnellement et socialement acceptable. Le cas de Mathilde (39 ans) illustre bien cette situation; "Ce n'est pas le côté financier qui compte hein... je veux dire oui j'avais des soucis d'argent (suite à ma séparation!), mais j'avais encore mon travail et j'étais très, très bien dans mon travail, cette reconnaissance que l'on a au travail est très importante, plus même que le côté financier».

A l'inverse, ce sont les personnes au chômage qui sont les plus fragilisées. Pour de nombreux répondants, l'exclusion de la sphère de la consommation des biens et services va de pair avec l'exclusion du marché du travail. Cela entraîne des possibilités limitées d'interaction sociale, une tendance à l'exclusion des groupes affinitaires et un sentiment d'isolement, comme l'exprime si bien Laurent (40 ans) : "Là y a carrément un phénomène psychologique que je ne saurais pas expliquer, je ne suis pas psychologue mais y a un repli sur soi-même comme une bête qui se terre dans son terrier quoi;... c'est un peu gênant par rapport à vos amis qui eux travaillent, qui ont les moyens...".

On retrouve ici un enchâssement de différents types de précarités (touchant au travail, à la sphère sociale et à la consommation) qui fragilise encore plus les individus et tend à les installer plus durablement dans la pauvreté. Ces pratiques rapportées par les répondants sont à mettre en perspective avec les résultats de George et Maddox (1977) selon lesquels les individus confrontés au chômage se retrouvent brusquement contraints à mener une vie totalement différente. Ils ont beaucoup plus de temps libre, n'ont plus cette reconnaissance qu'ils avaient lorsqu'ils étaient à leurs postes de travail, subissent une pression sociale stigmatisante et sont confrontés à une perte de confiance en soi qui rend la situation plus difficile à gérer.

\section{EMANCIPATION PROGRESSIVE DES CONTRAINTES DU SYSTÈME MARCHAND}

Durant la transition par l'épisode de pauvreté, les individus expérimentent des tentatives de «sortie» du marché comme réponse à la détérioration de leur situation financière et sociale. Cette «sortie» du système marchand prend des formes temporaires circonscrites à la phase de liminalité (i), ou tend à s'ancrer de manière plus durable et choisie dans la phase d'agrégation (ii). 


\section{Les stratégies d'ajustement temporaires en phase de liminalité}

Les consommateurs interrogés déploient entre autres mesures pour ajuster leur consommation à la contrainte de restriction financière, le report d'achats planifiés. Cette tendance à la procrastination n'a pas été identifiée auparavant comme une stratégie de coping avec les difficultés financières rencontrées par les individus structurellement pauvres dans la mesure où les perspectives de sortie n'y sont pas envisagées. Il ressort ainsi que des informants, arrivent, peu ou prou, à consommer mais en s'extrayant de l'immédiateté et du rythme imposés par le marché ou la pression sociale, en ne cherchant pas à satisfaire leurs besoins dès lors qu'ils sont éprouvés, mais en différant les réponses dans le temps.

Ce faisant, ces consommateurs tendent à se consoler en anticipant un avenir meilleur en se projetant notamment en sortie de crise : "»Je me suis privé de certaines choses, donc euh par exemple euh... il y'avait un jeu que j'attendais impatiemment et je n'ai pas pu me permettre de l'acheter lorsqu'il est sorti j'ai dî attendre quelques mois pour l'acheter, je me suis dit ce n'est pas grave si j'attends" (Yoann, 29 ans). Cette manière détournée et bricolée de résister à la séduction de la consommation est à rapprocher de ce que Moisio et Aaskegard (2002) qualifient de détournement des codes de la consommation.

Une autre stratégie mise en place pendant la phase de restriction financière consiste pour les consommateurs à recourir à l'évitement comme moyen de résistance à soi et aux sirènes de la consommation. L'évitement peut concerner certains lieux d'achat ou certains rayons dans le magasin en vue de limiter la probabilité d'être confronté à une offre attrayante et de risquer de céder à l'appétit de consommer; "Bah du coup je consommais selon mes moyens, c'est à dire qu'avant je pouvais consommer sans réfléchir sauf que aujourd'hui, je consomme selon le budget que j'ai, au lieu de me laisser un peu tenter par certaines choses, bah non je me limitais aux choses qui étaient importantes et donc j'évitais de passer par les rayons où je savais pertinemment que j'allais être attirée par certains produits" (Catherine, 28 ans).

Ces stratégies de report d'achat et d'évitement déployées par les individus constituent des formes modérées et ponctuelles d'émancipation du marché et de résistance à la consommation (Penaloza; Price, 1993). Elles traduisent la capacité de ces personnes à puiser dans leurs propres ressources (en l'occurrence psychologiques) pour discipliner leur comportement de consommation et l'ajuster à la situation de restriction financière qu'ils traversent. Ces stratégies d'adaptation s'accompagnent d'arrangements identitaires opérés par les individus en vue de se soustraire à la consommation. Cela se traduit par une domestication identitaire consistant à se faire violence pour se défaire de ses habitudes d'achat et de ses relations aux marques en vue de se définir autrement en consonance avec la «culture du pauvre».

\section{Les pratiques durables d'émancipation en phase d'agrégation}

Les individus ayant vécu une expérience de restriction financière avec son lot de frustrations et de privations, n'en sortent généralement pas indemnes quand ils retrouvent une situation socio-économique plus favorable à l'issue de l'épisode de pauvreté. Ainsi, certains consommateurs ont continué à limiter leur consommation à la sortie de la phase de restriction alors qu'ils ont retrouvé un statut plus propice à la consommation. La période de restriction financière a ainsi été l'occasion pour eux de s'éveiller et d'adhérer à de nouvelles valeurs de consommation raisonnée et de simplicité volontaire (Elgin, 1981). Leurs motivations de consommation post-restriction deviennent plus idéologiques et les amènent à mieux contrôler leurs comportements et à s'inscrire dans une logique d'émancipation assumée du système marchand. On assiste ainsi à une prise de position beaucoup plus appuyée qui s'exprime à travers un discours critique à l'égard de la consommation, donnant à voir l'émergence d'une figure de militantisme anticonsumériste. Le niveau de cet affranchissement varie des simples cas de prise de conscience de la possibilité de consommer autrement en adoptant des circuits et modes alternatifs à l'instar de Philippe (45 ans) qui dit; "Je me suis rendu compte qu'on pouvait très bien vivre avec peu et bien, consommer ailleurs, sans pour autant avoir une frénésie de la consommation "; aux situations où l'individu est beaucoup plus impliqué et assume sa prise de position critique par rapport au système marchand : "Je suis contre cette idéologie de la valeur de la consommation, même si ça a de l'importance dans la société actuelle ... c'est quelque chose à la fois dont je suis consciente et à la fois je suis vachement rebelle... parce que je me dis mais la consommation c'est une négation des véritables rapports humains, on t'aime pas parce que aujourd'hui tu as une veste de telle marque ou parce que tu bouffes des germes bio quoi, tu vois c'est pas ça..." (Bérénice, 25 ans). Le fait d'être dans l'obligation de limiter leur consommation pendant la phase de restriction des revenus a fait prendre conscience aux individus de la pression que véhicule insidieusement la société de consommation en termes de normes : "... le fait que je doive assez souvent me fixer des limites dans ma consommation quand je sors avec des gens... ces choses-là oui je sens que la plupart des autres personnes ne le font pas forcément et donc ça m'a fait prendre conscience que la société de consommation et bien ça me dégoûte et il faudrait que ça cesse; mais ça ne cessera pas car les gens sont comme ça, les gens c'est des pigeons" (Maiwen, 24 ans).

En outre, l'expérience de restriction des revenus a permis aux individus de s'approprier des pratiques de consommation qui leur semblaient dévalorisantes avant (telles que la récupération d'objets ou de produits alimentaires mis au rebus par d'autres) et de se redéfinir identitairement en conséquence : «La restriction ne m'a pas amené à des pratiques euh telles que, la récup ou des choses comme ça parce que je vivais déjà tellement mal le fait d'avoir un budget restreint que pour moi les récupérer ou faire les fins de marchés c'était la honte suprême; alors que maintenant je les considère plus du tout pareil... mais c'était la hoooooonte, c'est parce que je les voyais pas comme 'pourquoi payer plus cher', je le voyais comme... euh... j'ai pas le choix, je suis obligé de faire ça... ça c'était très dur... Donc du coup je ne pouvais pas trop m'ouvrir sur des méthodes alternatives alors que maintenant ça fait partie des choses que je fais avec beaucoup de plaisir» (Théophile, 28 ans). Cette forme d'émancipation des normes de consommation apparaît de manière assumée postépisode de pauvreté et rend compte de la tension vécue pendant la phase de transition liminale lorsque l'individu se voyait contraint d'adopter ces pratiques pour (sur)vivre. Cette tension résulte de l'opposition entre la réalité du pauvre que vivent ces individus et la culture matérielle qui les entoure (Hill, 2008). 
Dans ces deux stratégies ponctuelle et durable d'affranchissement des normes de consommation, le coping revêt une forme «d'ajustement progressifen réaction à une situation d'influence perçue «que l'on peut mettre en perspective avec les éléments définitoires de la résistance du consommateur (Roux, 2007). C'est essentiellement lors de la transition entre les phases de liminalité et d'agrégation, qu'une forme de résistance «à» la consommation se met graduellement en place. Celle-ci débute par une phase d'évitement et de report d'achat pour évoluer ensuite vers des logiques d'opposition plus engagées menant à la réduction de la consommation comme un choix de vie (Elgin, 1981) et à la rébellion comme mode d'action contre le marché (Ritson; Dobscha, 1999). Ces deux modes de comportements expriment un militantisme individuel qui vise à s'émanciper de la culture matérielle. Par conséquent, une restriction des ressources subie temporairement peut se transformer en une réduction choisie de la consommation post épisode de pauvreté, en l'occurrence en un acte assumé vecteur d'un nouveau mode de vie critique et protestataire.

Sur le plan des arrangements identitaires auxquels sont confrontés les individus qui s'inscrivent dans une logique d'émancipation des normes du marché, l'expérience de restriction des ressources génère une prise de conscience de la futilité de la consommation et produit des actes «hérétiques» de rejet ou de rébellion à l'encontre des pratiques marketing et du système marchand (Ritson; Dobscha, 1999). Ces changements identitaires se manifestent ici de manière individuelle sous la forme d'actes d'opposition militants, d'endossement d'identités anti-culture de consommation et de dissidence par rapport aux normes du marché. Cela exprime une sorte de contestation du pouvoir du marché et d'empowerment des individus entendu comme «un état mental qui s'accompagne souvent d'un acte physique permettant à des consommateurs d'opérer leurs propres choix et d'exprimer de manière active leurs propres volontés dans leur prise de décision au lieu de se soumettre à ce qui est offert ou attendu par les firmes» (Wright et al. 2006).

\section{Pratiques de Consommation déviantes et RisQue de MARGINALISATION}

Si la période de restriction financière est l'occasion d'une prise de conscience du sens de la consommation, elle est aussi à l'origine de l'émergence de comportements d'achat et de consommation déviants par rapport à la norme (Amine; Gicquel, 2011). La déviance peut être définie comme «le produit d'une transaction effectuée entre un groupe social et un individu qui, aux yeux du groupe, a violé une norme» (Becker, 1963). Au niveau du marché, certains individus n'ayant pas les moyens de se conformer aux normes de consommation résolvent ce dilemme par l'adoption de pratiques déviantes.

Ainsi, certains informants ont manifesté des formes de consommation déviante générée par le contexte de baisse des ressources financières qu'ils ont vécu. Ces comportements «anormaux» prennent des formes et des intensités variables, allant d'une simple intention préalable au passage à un acte délictueux à l'instar de Maiwen (24 ans) qui raconte : «si jamais j'étais dans le besoin plus que ça, j'aurai certainement pas de scrupule à avoir ce comportement là avec les grands magasins supermarché ou les grandes marques, j'aurai tendance à me dire ces gens-là se font beaucoup plus d'argent que ce qu'ils devraient, ça serait donc pas mal d'équilibrer ça en leur piquant des choses "; en passant par une déviance tempérée dans le sens où elle renvoie à des actions (et non plus des intentions) qui sortent du cadre des règles et conventions sociales comme c'est le cas de Camille (31 ans) : "Je me suis retrouvée un dimanche à marcher dans la rue, cherchant les mégots de cigarettes par terre parce que je n'avais plus du tout de clopes «; pour arriver enfin à une déviance extrême qui traduit des comportements répréhensibles par la loi comme l'illustre Bérénice (25 ans) : «Des fois, il m'est arrivé de ne plus avoir un rond, alors j'ai... j'ai volé, je pense qu'on a tous volé un jour dans notre vie, enfin j'ai volé de la nourriture dans les supermarchés; style fromage, jambon, fruits...».

Pour l'ensemble des informants qui les ont exprimés, ces comportements déviants ont un caractère temporaire circonscrit à la durée de la restriction des ressources. En effet si les individus s'autorisent à transgresser la norme pendant la phase de restriction financière, ils tendent en général à la réintégrer dès lors que cette phase est dépassée. Il en résulte que les individus tendent à ne pas s'accommoder durablement des pratiques déviantes en raison du risque de marginalisation et de sanction, et cherchent à revenir vers la norme du groupe social. Ces comportements déviants prennent la forme «d'actes non-fortuits opérés au service d'une fin» (Cusson, 1992), en l'occurrence celle de dégager des marges de manœuvre en matière d'arbitrage des dépenses et/ou de gestion du soi social en maintenant une consommation jugée inclusive.

La phase de restriction financière a eu cependant d'autres répercussions négatives du point de vue social. Les individus qui se sont renfermés sur eux-mêmes ont vu leurs rapports sociaux se dégrader petit à petit pour s'installer progressivement dans la marge et l'isolement à l'instar de Laurent (40 ans) : «... y a un repli sur soi même ..., et pour pas être un fardeau pour les autres, pour pas qu'ils aient une mauvaise image de vous quoi, vous n'allez plus les voir et voilà. Après vous vous en remettez et là vous n'avez plus d'amis ». La réduction des ressources favorise ainsi l'exclusion graduelle de l'individu du groupe social et l'inscrit dans une spirale de ruptures (familiale, sociale, de travail) qui le conduit inéluctablement à l'isolement et à la marginalisation. Elle finit par le convaincre d'internaliser ces conditions de pauvres qui le séparent des insiders, en l'occurrence ceux qui sont dans la société de consommation. On retrouve ici les ingrédients du déclassement social qui inscrit les individus de manière durable dans la pauvreté.

\section{LES VECTEURS D'INSTITUTIONNALISATION DE LA PAUVRETÉ}

A travers les discours des répondants, la durée de l'épisode de pauvreté paraît corrélée au nombre de transitions ou de ruptures successives ou simultanées par lesquelles passent les individus. Plus ces derniers doivent faire face à une pluralité de ruptures (travail, santé, logement, divorce) plus la durée de l'épisode est longue. Cette multiplicité de ruptures fragilise structurellement les individus et les rend encore plus vulnérables aux aléas de la vie pour finir par les installer durablement dans la pauvreté.

Deux profils des répondants se dégagent sur le plan de la durée de l'épisode de pauvreté et de son incidence sur le plan de la consommation et de la réévaluation identitaire. 
- La majorité des individus interrogés (neuf sur les quinze), sont passés par une seule transition (réduction des ressources). Pour ces informants, la période de baisse des ressources peut être qualifiée d'assez courte puisqu'elle dure un peu plus de trois mois en moyenne. Dans ce cas, la phase de liminalité est réduite et entrouvre un interstice permettant aux individus d'entrevoir assez rapidement l'après restriction et les perspectives de se reconstruire identitairement. Par ailleurs, au regard de la durée courte de la période de transition, la réévaluation des pratiques de consommation et des registres identitaires tend à être circonscrite et temporaire dans la mesure où les individus peuvent se projeter assez rapidement dans la réintégration d'un statut socio-économique favorable adossé à une identité désirée.

- Par contre, les interviewés qui sont passés par plusieurs ruptures (baisse des revenus, divorce, déménagement, perte d'emploi), l'épisode de pauvreté s'est étalé sur une période assez longue allant jusqu'à plus de deux ans. Dans ces cas, la phase de liminalité se prolonge et la redéfinition identitaire tarde à se cristalliser, les personnes étant d'abord en ballotage entre la séparation de l'ancien soi et l'intégration du nouveau soi non encore stabilisé. Ensuite, les perspectives de sortie devenant incertaines, les individus modifient leurs modes de consommation et procèdent à des ajustements identitaires qui les installent progressivement dans la culture du pauvre.

Lorsque l'épisode de pauvreté est court, les individus n'ont pas le temps d'internaliser les nouveaux modes de consommation «du pauvre» et dès la sortie de la période de restriction ils se remettent à consommer de plus belle et s'installent dans une parenthèse de consommation frénétique avant que celle-ci ne redevienne normale. Ainsi, certains de nos informants retrouvent leur appétit de consommation d'avant la période de précarité et tendent à prendre leur revanche sur la privation vécue durant la période d'abstinence de la consommation de certains produits et services; "je suis sortie de crise je profite, je me dis bon bah, avant je pouvais pas, maintenant je peux alors je m'en fous je me permets. Alors je résiste oui mais qu'en période de crise, quand je suis sortie de la crise c'était terminé, des fois j'oublie les moments difficiles et... comme j'ai pas pu me faire plaisir auparavant bah du coup j'en profite au moment venu. C'est pas très bien ce que je dis mais c'est la vérité malgré tout" (Catherine, 28 ans).

Il ressort ici que la consommation est vue comme une activité valorisée ou critiquée selon le contexte social, où les gens jugent les autres et se jugent eux-mêmes en fonction de leur mode de vie (Rassuli; Hollander, 1986). L'interaction avec le marché offre aux consommateurs des ressources qu'ils mettent à profit pour reconfigurer leur identité en vue d'afficher une image de soi socialement acceptable. Cela inscrit l'individu dans un jeu de négociation identitaire pour s'adapter aux différents contextes d'interactions sociales, et rend compte d'une plasticité de l'identité qui contraste avec l'idée d'une identité unique et figée. L'identité sociale est ainsi centrée sur la visibilité des biens et services de consommation utilisés comme des signes d'affiliation à un groupe de référence au statut valorisé (Baudrillard, 1998). La consommation peut, à cet égard, être vue comme un facteur de classification pour se rapprocher d'une identité désirée et socialement valorisée et/ou de différenciation sociale pour s'éloigner d'une identité du «pauvre» socialement honnie et stigmatisée.

En revanche, lorsque la période de restriction dure trop longtemps et que les individus s'y installent, ces derniers reconsidèrent leurs choix et voient se normaliser les caractéristiques de la «figure du pauvre» (difficulté de se projeter plus avant, altération de l'image de soi, stress, pessimisme, ...). En conséquence, la phase de restriction financière s'étalant dans le temps, les arbitrages fondés sur le prix se normalisent et ont tendance à le rester même après la sortie de crise car les individus prennent de nouvelles habitudes de consommation auxquelles ils deviennent attachés, comme l'exprime Bérénice (25 ans) : "Maintenant je consomme des marques de distributeurs j'ai réussi à passer ce cap ça me gêne pas du tout je l'assume complètement après coup. Imagine tu viens d'une famille où la qualité de la bouffe c'est... euh c'est l'oracle, c'est Dieu, c'est la religion; donc ça s'est fait progressivement en passant par des marques pour ensuite basculer vers des marques de distributeurs mais maintenant, ça ne me gêne pas du tout, du tout, du tout...».

En outre, ce sont les individus qui ont vécu la période de pauvreté la plus longue et qui avaient un niveau de vie initial élevé, qui ont vu leur identité se transformer de manière progressive à radicale. Si l'on suit les trajectoires de ces individus, nous constatons que pendant la phase de liminalité, ses derniers ont eu beaucoup de mal à accepter leur nouvelle situation. Ils ont été confrontés lors de l'épisode de pauvreté à d'importantes ruptures auxquelles ils ont dû s'adapter en s'appropriant les codes du nouveau groupe social. Cette évolution à terme vers l'intégration des codes de consommation des «pauvres» traduit une internalisation de ces modes de vie par les individus qui finissent par admettre leur nouvelle situation. Ce faisant, la perspective d'une sortie de crise et d'une amélioration potentielle de leurs conditions de vie s'éloigne (Yurdakul; Atik, 2016), de même que s'entrouvre en parallèle une manière frustrée et parfois revancharde de considérer la consommation et la culture matérielle.

\section{Implications de la recherche pour les entreprises, le marché et la société}

L'objectif de cette recherche était d'identifier les mécanismes d'adaptation mobilisés par les individus lorsqu'ils traversent des périodes de baisse temporaire des ressources et l'influence de cette expérience à la fois sur les pratiques de consommation et sur les ajustements identitaires à l'œuvre. Les implications de cette recherche se déclinent sur les plans théorique, managérial et sociétal et ouvrent la voie à l'exploration de solutions innovantes et alternatives au marché en tant que leviers de réduction de la pauvreté et d'amélioration du bien-être des individus (Oosterlynck et al. 2013).

Au niveau théorique, une mise en perspective des résultats de la recherche avec la littérature montre un premier apport singulier dans le sens où la conception antérieure du consommateur pauvre comme un individu qui manque de ressources économiques et sociales ou qui est incapable de les mobiliser semble, de par sa fixité, davantage caractériser le "pauvre chronique» et s'avère inappropriée pour rendre compte du consommateur "pauvre transitoire». En effet, nos résultats donnent à voir une dynamique qui anime ce dernier l'amenant selon la phase de 
transition à résister, ajuster, accepter puis rejeter les conditions du pauvre. Il déploie parfois avec inventivité, des ressources personnelles, sociales ou puisées dans le marché pour adapter ses comportements de consommation et procéder à des arrangements identitaires en vue de résoudre les problèmes d'exclusion du groupe social et de la sphère marchande qu'il rencontre. Cette plasticité dans les pratiques et dans la configuration de soi dont font preuve les individus transitant par un épisode de pauvreté montre une réalité multiple et complexe du pauvre transitoire qui contraste et enrichit la vision monolithique de la figure du pauvre typique des travaux antérieurs.

Ensuite, tout en soulignant l'apport fécond du cadre théorique des transitions liminales à la compréhension des logiques d'ajustement de la consommation et de l'identité des individus en période de restriction financière, ce travail participe à l'enrichissement du corpus théorique sur les dispositifs de coping à travers l'identification de stratégies inédites mobilisées par ces individus. Ainsi, contrairement à ce qui caractérise la pauvreté durable, les arbitrages effectués par les personnes transitant par des épisodes de pauvreté se singularisent sur le plan de la consommation par des logiques de procrastination, d'émancipation, de résistance, voire de déviance et traduisent sur le plan identitaire des logiques de préservation ou de réévaluation de soi variables en fonction de la phase de transition. Nos résultats montrent notamment que les individus qui traversent une période de baisse des revenus mobilisent une palette variée de stratégies d'ajustement des comportements qui les installent tour à tour dans la figure (i) du consommateur qui cherche à éviter la stigmatisation et l'exclusion de la sphère de la consommation dans ses diverses dimensions utilitaires, hédoniques et symboliques (avec comme champ d'action le marché), (ii) du militant disposé à agir pour transformer et faire évoluer la société en s'émancipant des règles du marché, à contester les logiques de domination du système marchand et à vouloir instaurer un ordre économique et social alternatif (avec comme espace le champ politique et idéologique) et enfin (iii) du citoyen qui s'inscrit dans une logique de régénération des élans de solidarité et de retissage du lien social (avec comme périmètre la vie en collectivité).

Au niveau du marché, les consommateurs qui traversent une période de baisse de ressources financières constituent un segment de plus en plus large disposant d'un pouvoir d'achat certes altéré mais économiquement significatif à un niveau agrégé et justifiant une adaptation des stratégies marketing des firmes à cette base de la pyramide (Prahalad; Hart, 2002), y compris dans une logique inclusive. Cette sous-population ouvre des perspectives intéressantes pour (re)dynamiser les circuits alternatifs qui offrent d'appréciables rapports qualité/ prix ou qui valorisent des rapports humains plus authentiques (marchés de l'occasion, circuits courts, C2C) en particulier pour ceux qui s'inscrivent dans des «carrières » de simplistes volontaires. En s'installant dans des logiques de modération de leurs besoins via l'adoption de pratiques de consommation sobres (Rabhi, 2010), ces individus rompent avec l'ordre anthropophage instauré par la société de consommation et s'engagent dans des modes de consommation et de production plus responsables qui gagneraient à être davantage soutenus par les pouvoirs publics et les initiatives citoyennes.
Par ailleurs, la baisse temporaire des revenus des consommateurs interroge certaines pratiques commerciales des entreprises (type abonnements et engagements sur des durées assez longues) taillées sur des situations financières pérennes des clients, qui doivent être revues en vue de permettre à ces individus fragilisés de pouvoir basculer sur des offres plus adaptées à leur situation sans charges supplémentaires. Ces entreprises y gagneraient économiquement (baisse des ruptures de contrats, moins d'impayés, etc.) et participeraient par la même occasion à asseoir des relations marchandes stables tout en faisant preuve de responsabilité sociale.

Au niveau idéologique, la mise en place par les individus de logiques de résistance à la consommation par l'émancipation du système marchand et de déviance par l'affranchissement des normes, vise à se donner des latitudes d'arbitrage plus conséquentes et à s'ouvrir des interstices de confort dans la consommation et dans la définition de soi. L'émancipation du marché et de ses règles peut être vue comme une opportunité et non seulement une contrainte à la fois pour la sphère marchande et pour la société. Cette perspective, qui favorise l'émergence de comportements critiques à l'égard de la société de consommation est probablement salutaire à la fois pour un système de consommation à bout de souffle sur le plan écologique et de la gestion des ressources (réduction de la consommation, des déchets et des émissions) et pour régénérer ou explorer d'autres modes de production et de consommation de substitution (circuits courts, consommation biologique, autoconsommation, achats d'occasions et recyclage des objets). Cette évolution, potentiellement génératrice de valeur et d'emploi, est à soutenir absolument par les pouvoirs publics à travers des dispositifs proactifs accompagnant les nouvelles activités ainsi générées.

Par ailleurs, l'émancipation des normes du marché et de la culture matérielle pendant et à la sortie de l'épisode de pauvreté prend une forme de responsabilité individuelle qui préfigure des transformations significatives à venir au niveau de la société dans son ensemble. En adoptant, voire prônant, un mode de vie fondé sur la simplicité volontaire et la sobriété dans la consommation et par le rejet du modèle de la consommation à outrance érigé comme norme, ce comportement militant qui passe par le fait de discipliner d'abord sa propre consommation et celle de son entourage (volet prégnant dans cette recherche) avant de chercher à diffuser ces pratiques en "évangélisant » les autres (Kozinets; Handelmann, 2004) vise à transformer la société, en faisant évoluer à la fois les pratiques des individus et des acteurs au pouvoir (en pesant sur l'arsenal législatif pour mieux protéger les consommateurs et l'environnement et mieux responsabiliser des entreprises). Ces pratiques militantes constituent aussi à la fois une forme de résistance à la société de consommation (Roux, 2007) et une contestation des différentes formes de domination que produit le marché (Touraine, 1978). Elles peuvent être lues comme des logiques de contre-pouvoirs visant soit à créer des changements en restant dans le marché, soit à s'en affranchir en proposant de nouveaux modes de vie et de consommation alternatifs.

Au niveau sociétal, la mobilisation des relations sociales comme un amortisseur de la chute durant l'épisode de pauvreté traduit l'importance de la recherche de lien social et de solidarité à la fois familiale et de l'entourage dans la reconstruction 
identitaire des personnes touchées et dans leur recouvrement de la capacité de rebondir sur le plan socio-économique. Par conséquent la favorisation et l'organisation de la solidarité dans une logique citoyenne pour venir en aide aux individus traversant une période de difficulté financière (passagère ou longue) devient une nécessité en vue de (re)construire du lien là où les relations sociales ont tendance à se distendre sous l'effet de l'individualisme et sous l'assaut des différentes ruptures subites d'ordre familial, économique et social rencontrées par les individus.

A cette fin, des leviers alternatifs de lutte contre la pauvreté venant moins de la sphère économique classique que de la sphère sociale et associative (Laville, 2011; Oosterlynck et al. 2013) doivent être activés et articulés autour de l'économie sociale et solidaire, vue comme un nouveau modèle de société vecteur de réduction de la pauvreté des individus et d'impulsion de bienêtre ou a minima de mieux-vivre. Cette économie alternative se matérialise par une dynamique de la société civile et un essor des associations, coopératives et autres formes de regroupements alternatifs au marché qui investissent différentes sphères de la vie économique et sociale. En contribuant à insérer des individus traversant un épisode (plus ou moins long) de pauvreté dans l'économie sociale et solidaire, ce nouveau modèle est porteur des germes d'inclusion dans le champ de la consommation, de re-création de liens sociaux défaits, de réhabilitation identitaire des individus (en en faisant des acteurs utiles et non des assistés), mais aussi d'éducation aux valeurs de solidarité, de partage et de bien-être commun.

Une autre piste versant dans la même direction consiste pour les pouvoir publics et les établissements de crédits (notamment les banques mutualistes) à favoriser le développement du microcrédit en direction des personnes exclues du système bancaire en vue de combler l'absence de prêteurs classiques, à faciliter l'accès aux biens et services à la base de la pyramide (Fairbourne et al.2007) et à soutenir une sorte d'entreprenariat de nécessité (Tessier-Dargent; Fayolle, 2016).

Enfin, la durée de l'épisode de pauvreté constitue un risque d'institutionnalisation de la pauvreté contre lequel il convient impérativement de lutter. A la baisse des ressources financières s'ajoutent généralement des ruptures sur le plan familial (divorce, séparation), de la santé (détérioration) et du logement (perte du domicile), qui finissent par annihiler la capacité de résilience de l'individu. L'allongement de l'expérience de précarité produit un basculement de l'individu dans la pauvreté chronique et une internalisation du déclassement. L'une des responsabilités des pouvoirs publics et des milieux associatifs est de fonctionner en vigie sociale et d'intervenir assez tôt en vue de casser cette spirale dans laquelle risquent de s'installer les individus qui transitent par un épisode de pauvreté afin qu'ils retrouvent assez rapidement les ressorts nécessaires pour rebondir sur le plan économique et social et se reconstruire sur le plan identitaire.

\section{Bibliographie}

Amine, Abdelmajid; GicQuel, Yohan (2011). «Rethinking resistance and anti-consumption in the light of the concept of deviance", European Journal of Marketing, Vol. 45, N 11/12, p. 1809-1819.
BAUdrillard, Jean (1988). Consumer Society, in Poster M. (ed.), Jean Baudrillard: Selected Writings. Cambridge : Polity Press.

Bauman, Zygmunt (2005). Work, Consumerism and the New Poor, 2nd edition, Open University Press, Buckingham.

Becker, Howard S. (1963). Outsiders. Études de sociologie de la déviance, Paris : Editions Métailié.

BeLK, Russell (1988). «Possessions and extended self», Journal of Consumer Research, Vol.15, p. 139-168.

CAмвоis, Emmanuelle (2004). «Les personnes en situation difficile et leur santé», Les travaux de l'Observatoire national de la pauvreté et de l'exclusion sociale 2003-2004, ONPES, Paris : La Documentation française.

CaPlovitz, David (1963). The poor pay more: Consumer practices of low income families, New York : Free Press of Glencoe.

CAstel, Robert (1991) «De l'indigence à l'exclusion, la désaffiliation ", dans Donzelot J. et al., Face à l'exclusion, le modèle français. Paris : Éditions Esprit, Paris, p. 137-168.

Castel, Robert (1995). Les Métamorphoses de la question sociale. Une chronique du salariat. Paris : Fayard.

Couret, Dominique; Metzger, Pascal (2009). « Réduire les vulnérabilités plutôt qu'éradiquer la pauvreté», Espace, Populations, Sociétés, $\mathrm{N}^{\circ} 2$, p. 263-277.

Coyne, James C.; Aldwin, Caroline M.; Lazarus, Richard S. (1981). «Depression and coping in stressful episodes», Journal of Abnormal Psychology, Vol. 90, $\mathrm{N}^{\circ}$ 5, p. 439-447.

Cusson, Maurice (1992). Déviance, dans Boudon R. (dir.), Traité de sociologie, Paris : PUF.

Duhachek, Adam (2005). "Coping : A Multidimensional, Hierarchical Framework of Responses to Stressful Consumption Episodes", Journal of Consumer Research, Vol. 32, N² 2, 41-53.

ElgIN, Duane (1981). Voluntary Simplicity: Toward a Way of Life that is Outwardly Simple, Inwardly Rich. New York: William Morrow and Company.

Elliott, Richard; Leonard, Claire (2004). «Peer Pressure and Poverty: Exploring fashion brands and consumption symbolism among children of the British Poor», Journal of Consumer Behaviour, Vol. 4, No 3, p. 347-359.

Fairbourne, Jason S.; Gibson, Stephen W.; Dyer, W Gibb (Eds.) (2007). Microfranchising: Creating wealth at the bottom of the pyramid. Edward Elgar Publishing.

Favreau, Louis (2003). «Développement local et lutte contre les inégalités : de quelques innovations québécoises (1980-2000)", Revue Interventions Economiques, 30, p. 1-20.

Fraisse, Laurent; Guérin, Isabelle; LAVILle, Jean-Louis (2007). «Économie solidaire: des initiatives locales à l'action publique. Introduction", Revue Tiers Monde, Vol. 2, N 190, p. 245-253.

GEORGE, Linda K.; MADDOx, George L. (1977). «Subjective adaptation of the loss of the work role : A longitudinal study». Journal of Gerontology, Vol. 32, № 4, p. 456-462.

Goffman, Erving (1973). La mise en scène de la vie quotidienne. Tome I: La présentation de soi. Paris : Éditions de Minuit.

Gorge, Hélène; Ozçaglar-Toulouse, Nil (2013). «Expériences de consommation des individus pauvres en France : Apports du Bas de la Pyramide et de la Transformative Consumer Research », Décisions Marketing, Oct.-Déc., N 72, p. 139-156. 
HalbWachs, Maurice (1994). Consommation et société, Paris : PUF.

Hamilton, Kathy; Catterall, Miriam (2006). «Transitions into poverty: An exploratory study into how families cope when faced with income reduction and limited consumption opportunities», Marketing Review, Vol. 6, N² 2, p. 123-136.

Hill, Ronald P. (2008). «Disadvantaged Consumers : An Ethical Approach to Consumption by the Poor ", Journal of Business Ethics, Vol. 80, p. 77-83

Hoge, Dean R.; Mc Carthy, John D. (1984). «Influence of individual and group identity salience in the global self-esteem of youth », Journal of Personality and Social Psychology, Vol. 47, p. 319-340.

Hulme, David; Shepherd, Andrew (2003). «Conceptualizing Chronic Poverty», World Development, Vol. 31, No 3, p. 403-424.

Kozinets, Robert V.; Handelman, Jay M. (2004). «Adversaries of Consumption : Consumer Movements, Activism, and Ideology", Journal of Consumer Research, Vol, 31, № 3 , p. 691-704.

LAVILLE, Jean-Louis (2011). «De l'indignation à un nouveau projet politique», Alternatives économiques.fr, 21 Novembre 2011.

LAZARUS, Richard S. (1966). Psychological stress and the coping process, New York : Mc Graw-Hill.

LoIsy, Christian (2000). «Pauvreté, précarité, exclusion. Définitions et concepts", Les Travaux de l'Observatoire de la DREES, Cahier $\mathrm{N}^{\circ} 1$, p. 23-52.

MArris, Peter (1974). Loss and Change, London : Routledge \& Kegan Paul.

McKay, Andrew; Lawson, David. (2003). «Assessing the extent and nature of chronic poverty in low income countries : issues and evidence», World Development, Vol. 31, No 3, p. 425-439.

Mini-dossiers «Pauvreté, précarité et consommation» (2014/2015). Revue Economies et Sociétés, Série "Etudes critiques en manage-

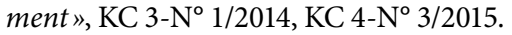

Moisio, Risto; AskegaARD, Soren (2002). «Fighting culture. Mobile phone consumption practices as means of consumer resistance", Asia Pacific Advances in Consumer Research, ACR, Vol. 5, p. 24-29.

Morin, Violette (1969). «L'objet biographique», Communications, Vol. 13, No1, p.131-139.

Noble, Charles H.; WALKer, Beth A. (1997). «Exploring the relationships among liminal transitions, symbolic consumption, and the extended self", Psychology and Marketing, Vol. 14, No 1 , p. 29-47.

Oosterlynck, Stijn; Kazepov, Yuri; Novy, Andreas; Cools, Pieter; BArberis, Eduardo; Wukovitsch, Florian; SARIUs, Tatiana; LEubolt, Bernhard (2013). "The butterfly and the elephant: local social innovation, the welfare state and new poverty dynamics », ImPRovE Working Paper, $\mathrm{N}^{\circ}$ 13/03, April, Antwerp, 45p.
Paugam, Serge (2005). Les formes élémentaires de la pauvreté, Paris : PUF.

Paugam, Serge; Seltz, Marion (2005). «La perception de la pauvreté en Europe depuis le milieu des années 1970 », Economie et Statistique, No 383-384-385, p. 283-305.

Peñaloza, Lisa; Price, Linda L. (1993). «Consumer resistance : a conceptual overview», Advances in Consumer Research, McAlister L. and Rothschild M.L. eds., ACR, Vol. 20, p.123-128.

Prahalad, Coimbatore K.; Hart, Stuart L. (2002). «The fortune at the bottom of the pyramid», Strategy and Business, $\mathrm{N}^{\circ} 26$, p. 1-14.

RabHI, Pierre (2010). Vers la sobriété heureuse, Arles : Actes Sud.

Rassuli, Kathleen M.; Hollander, Stanley C. (1986). «Desire Induced, Innate, Insatiable?», Journal of Macromarketing, Vol. 6, p. 4-24.

Ritson, Mark; Dobscha, Susan (1999). «Marketing Heretics : Resistance is/is not futile», Advances in Consumer Research, Arnould E. and Scott L. eds., ACR Vol. 26, p. 159.

Roux, Dominique (2007). «La résistance du consommateur : Proposition d'un cadre d'analyse», Recherche et Applications en Marketing, Vol. 22, $\mathrm{N}^{\circ} 4$, p. 59-80.

SEn, Amartya (1985). Commodities and Capabilities, Oxford : Elsevier Science Publishers.

Schouten, John W. (1991). «Selves in transition : symbolic consumption and personal rites of passage and identity construction ", Journal of Consumer Research, Vol. 17 (March), p. 412-26

Simmel, Georg (1907/1998). Les pauvres, Paris : PUF.

Tessier-Dargent, Christel; Fayolle, Alain (2016). «Une approche typologique de l'entrepreneuriat de nécessité ", Revue Interdisciplinaire Management, Homme et Entreprise, Vol. 3, $\mathrm{N}^{\circ} 22,74-92$.

Toumi, Alia (2015). «Pauvreté permanente vs pauvreté transitoire: conceptualisation et exploration de leurs effets en termes d'ajustement de la consommation", Economies et Sociétés, Série "Etudes critiques en management", KC N ${ }^{\circ} 4 / 2$, Février, p. 275-295.

Touraine Alain (1978). La Voix et le Regard. Sociologie des mouvements sociaux, Paris : Editions Seuil.

VAn GenneP, Arnold (1909/1981). Les rites de passage, Paris : Picard.

Wright, Len; Newman, Andrew; Dennis, Charles (2006). "Enhancing consumer empowerment», European Journal of Marketing, Vol. 40, № 9/10, p. 925-935.

Yurdakul, Dicle; Atik, Deniz (2016). «Coping with Poverty through Internalization and Resistance : The Role of Religion", Journal of Macromarketing, Vol. 36, N³, p. 321-336. 


\begin{tabular}{|c|c|c|c|c|c|}
\hline \multicolumn{6}{|c|}{$\begin{array}{l}\text { ANNEXE } 1 \\
\text { Profils des répondants }\end{array}$} \\
\hline Individus & Sexe & Age & $\begin{array}{l}\text { Profession actuelle (durant } \\
\text { l'épisode de pauvreté) }\end{array}$ & Type et durée de l'entretien & Types de transition \\
\hline Catherine & $\mathrm{F}$ & 28 & $\begin{array}{l}\text { Femme au foyer } \\
\text { (sans emploi durant l'épisode } \\
\text { de pauvreté) }\end{array}$ & $\begin{array}{l}\text { Rétrospectif } \\
\text { (post restriction financière) } \\
\text { (6 mois) }\end{array}$ & $\begin{array}{l}\text { A connu une période de pauvreté et s'en } \\
\text { est sortie larrivée d'un nouveau-nél }\end{array}$ \\
\hline Alexandre & $\mathrm{H}$ & 25 & $\begin{array}{l}\text { Etudiant stagiaire } \\
\text { létudiant durant l'épisode } \\
\text { de pauvretél }\end{array}$ & $\begin{array}{l}\text { Rétrospectif } \\
\text { (post restriction financière) } \\
\text { ( } 3 \text { mois) }\end{array}$ & $\begin{array}{l}\text { A connu un épisode de pauvreté et s'en } \\
\text { est sorti (départ du domicile parental - } \\
\text { prise d'autonomie) }\end{array}$ \\
\hline Théophile & $\mathrm{H}$ & 28 & $\begin{array}{l}\text { Chargé d'études } \\
\text { (sans emploi durant l'épisode } \\
\text { de pauvreté) }\end{array}$ & $\begin{array}{l}\text { Rétrospectif } \\
\text { (post restriction financière) } \\
\text { (20 mois) }\end{array}$ & $\begin{array}{l}\text { A connu une période de pauvreté et s'en } \\
\text { est sorti (chômage + décès du père) }\end{array}$ \\
\hline Laurent & $\mathrm{H}$ & 40 & $\begin{array}{l}\text { Professeur } \\
\text { (sans emploi durant l'épisode } \\
\text { de pauvreté) }\end{array}$ & $\begin{array}{l}\text { Rétrospectif } \\
\text { (post restriction financière) } \\
(2 \text { mois })\end{array}$ & $\begin{array}{l}\text { A connu un épisode de pauvreté et s'en } \\
\text { est sorti (chômage) }\end{array}$ \\
\hline Camille & $\mathrm{F}$ & 31 & $\begin{array}{l}\text { Architecte } \\
\text { (stagiaire durant l'épisode } \\
\text { de pauvreté) }\end{array}$ & $\begin{array}{l}\text { Rétrospectif } \\
\text { (post restriction financière) } \\
\text { (24 mois) }\end{array}$ & $\begin{array}{l}\text { A connu une période de pauvreté et s'en } \\
\text { est sorti (prise d'autonomie + baisse } \\
\text { des revenus) }\end{array}$ \\
\hline Bérénice & $\mathrm{F}$ & 25 & $\begin{array}{l}\text { Etudiante } \\
\text { létudiante durant l'épisode } \\
\text { de pauvretél }\end{array}$ & $\begin{array}{l}\text { Rétrospectif } \\
\text { (post restriction financière) } \\
\text { (12 mois) }\end{array}$ & $\begin{array}{l}\text { A connu un épisode de pauvreté et s'en } \\
\text { est sortie (changement de ville + prise } \\
\text { d'autonomie) }\end{array}$ \\
\hline Philippe & $\mathrm{H}$ & 45 & $\begin{array}{l}\text { Affaires étrangères } \\
\text { lactif durant l'épisode } \\
\text { de pauvreté) }\end{array}$ & $\begin{array}{l}\text { Rétrospectif } \\
\text { (post restriction financière) } \\
(7 \text { mois) }\end{array}$ & $\begin{array}{l}\text { A connu une période de pauvreté et s'en } \\
\text { est sorti (divorce) }\end{array}$ \\
\hline Maiwen & $\mathrm{H}$ & 24 & $\begin{array}{l}\text { Etudiante } \\
\text { létudiante durant l'épisode } \\
\text { de pauvretél }\end{array}$ & $\begin{array}{l}\text { Rétrospectif } \\
\text { (post restriction financière) } \\
\text { ( } 2 \text { mois) }\end{array}$ & $\begin{array}{l}\text { A connu un épisode de pauvreté et s'en } \\
\text { est sortie (changement de ville + prise } \\
\text { d'autonomie) }\end{array}$ \\
\hline Mathilde & $\mathrm{F}$ & 39 & $\begin{array}{l}\text { Secrétaire } \\
\text { lactive durant l'épisode } \\
\text { de pauvreté) }\end{array}$ & $\begin{array}{l}\text { Rétrospectif } \\
\text { (post restriction financière) } \\
\text { (24 mois) }\end{array}$ & $\begin{array}{l}\text { A connu une période de pauvreté et s'en } \\
\text { est sortie (divorce) }\end{array}$ \\
\hline Luc & $\mathrm{H}$ & 27 & $\begin{array}{l}\text { Etudiant } \\
\text { (actif durant l'épisode } \\
\text { de pauvreté) }\end{array}$ & $\begin{array}{l}\text { Durant l'épisode de pauvreté } \\
\text { (6 mois) }\end{array}$ & $\begin{array}{l}\text { Vit encore un épisode de pauvreté } \\
\text { (prise d'autonomie) }\end{array}$ \\
\hline Karen & $\mathrm{F}$ & 22 & $\begin{array}{l}\text { Etudiante active } \\
\text { létudiante sans emploi durant } \\
\text { l'épisode de pauvretél }\end{array}$ & $\begin{array}{l}\text { Durant l'épisode de pauvreté } \\
\text { (3 mois) }\end{array}$ & $\begin{array}{l}\text { Vit encore une période de pauvreté } \\
\text { (prise d'autonomie + déménagement } \\
\text { + chômage) }\end{array}$ \\
\hline Fred & $\mathrm{H}$ & 26 & $\begin{array}{l}\text { Etudiant } \\
\text { (étudiant durant l'épisode } \\
\text { de pauvreté) }\end{array}$ & $\begin{array}{l}\text { Durant l'épisode de pauvreté } \\
\text { (2 mois) }\end{array}$ & $\begin{array}{l}\text { Vit encore un épisode de pauvreté } \\
\text { (prise d'autonomie) }\end{array}$ \\
\hline Ludovic & $\mathrm{H}$ & 32 & $\begin{array}{l}\text { Cadre lactif durant l'épisode } \\
\text { de pauvretél }\end{array}$ & $\begin{array}{l}\text { Durant l'épisode de pauvreté } \\
\text { (2 mois) }\end{array}$ & $\begin{array}{l}\text { Vit encore une période de pauvreté } \\
\text { (baisse des revenus) }\end{array}$ \\
\hline Heykel & $\mathrm{H}$ & 26 & $\begin{array}{l}\text { Caissier à Carrefour lactif } \\
\text { durant l'épisode de pauvretél }\end{array}$ & $\begin{array}{l}\text { Durant l'épisode de pauvreté } \\
\text { (9 mois) }\end{array}$ & $\begin{array}{l}\text { Vit encore un épisode de pauvreté } \\
\text { (changement de ville + installation en } \\
\text { couple) }\end{array}$ \\
\hline Yoann & $\mathrm{H}$ & 29 & $\begin{array}{l}\text { Etudiant actif (étudiant } \\
\text { durant l'épisode de pauvretél }\end{array}$ & $\begin{array}{l}\text { Durant l'épisode de pauvreté } \\
\text { (4 mois) }\end{array}$ & $\begin{array}{l}\text { Vit encore une période de pauvreté } \\
\text { (prise d'autonomie) }\end{array}$ \\
\hline
\end{tabular}

